\title{
SATISFACCIÓN FAMILIAR, COMUNICACIÓN E INTELIGENCIA EMOCIONAL
}

\author{
Heriberto Rodríguez-Mateo \\ Departamento de Psicología, Sociología y Trabajo Social \\ Universidad de Las Palmas de Gran Canaria, España \\ heriberto.rodriguez@ulpgc.es \\ Isabel Luján Henríquez \\ Departamento de Psicología, Sociología y Trabajo Social \\ Universidad de Las Palmas de Gran Canaria, España \\ Carmen Delia Díaz Bolaños \\ Departamento de Psicología, Sociología y Trabajo Social \\ Universidad de Las Palmas de Gran Canaria, España \\ José Carlos Rodríguez Trueba \\ Departamento de Psicología, Sociología y Trabajo Social \\ Universidad de Las Palmas de Gran Canaria, España \\ Yurena González Sosa \\ Máster en Mediación e Intervención Familiar \\ Raquel Irene Rodríguez Rodríguez \\ Psicóloga. Máster en Mediación e Intervención Familiar
}

Fecha de Recepción: 11 Febrero 2018

Fecha de Admisión: 10 Abril 2018

\section{RESUMEN}

Para el desarrollo de las personas y de la sociedad en general, la institución familiar es un pilar fundamental. Una de las motivaciones sociales más importantes del ser humano desarrollada durante la infancia es la afiliación: mantener relaciones afectivas positivas con otras personas. Además, uno de los afectivos vitales en el ser humano es la relación de pareja, ya que permite satisfacer necesidades básicas y a su vez establecer pautas de desarrollo y comportamiento.

Basándonos en el modelo de inestabilidad familiar propuesto por Rodríguez, Rodríguez-Mateo y Luján (2015), el presente trabajo tiene varios objetivos: primero, conocer si las variables familiares interrelacionadas entre sí en dicho modelo (estrés familiar, comunicación familiar, los recursos familiares, satisfacción familiar e inestabilidad marital) pueden relacionarse con una nueva variable (la Inteligencia Emocional); y en segundo lugar, obtener posibles influencias e interacciones, tratando de replicar el modelo propuesto por dichos autores.

Esta investigación está centrada en la búsqueda de los distintos grados de asociación o relación que existe entre un grupo relevante de variables presentes en el entorno familiar con la satisfacción 


\section{SATISFACCIÓN FAMILIAR, COMUNICACIÓN E INTELIGENCIA EMOCIONAL}

y la inestabilidad matrimonial. Este conjunto de variables relacionadas con el ciclo vital familiar (comunicación familiar, recursos familiares, estrés familiar) afectan de forma directa a las relaciones familiares y se asocia a distintos grados de bienestar en las familias. Además, se ha añadido una nueva variable con respecto al estudio sobre el modelo de inestabilidad familiar en la ruptura de pareja (Rodríguez, Rodríguez-Mateo y Luján, 2015), la inteligencia emocional, para comprobar su relación con la estabilidad marital. A través de diferentes escalas se quiere evaluar dichas relaciones y comprobar si esos factores (estrés, comunicación, recursos e inteligencia emocional) inciden de forma directa sobre la satisfacción y la estabilidad familiar.

Los instrumentos utilizados en el presente trabajo de investigación, para operativizar las variables a estudiar, han sido recopilados del Manual de Instrumentos de evaluación familiar (2008) publicado por La Universidad de Deusto y la Escala de Inteligencia Emocional Autoinformada (TMMS-24) de Extremera y Fernández-Berrocal (2004).

La muestra corresponde a un grupo de 96 personas, cuyos criterios de inclusión fueron la pertenencia a grupo familiar y sin dificultad cognitiva para poder realizar los cuestionarios que se anexan.

Las técnicas de análisisde datos utilizadas han sido el análisis correlacional entre las variables familiares, la regresión lineal múltiple, análisis de propuesta de modelo estructural a través de la confirmación de las relaciones entre las variables (modelo confirmatorio), y el análisis factorial confirmatorio.

Se observó dos factores, la comunicación total y los recursos totales como variables predictoras de la satisfacción total. Se ha podido obtener un modelo confirmatorio que explica las variables asociadas a la satisfacción familiar a través del programa AMOS. Se sigue postulando, a través del análisis factorial confirmatorio, la variable vivencia familiar como "inyectora" del resto de variables.

Los resultados apoyan un nuevo modelo explicativo de relación entre dichas variables y confirman el poder predictivo de la comunicación y los recursos familiares en la satisfacción familiar.

Se pretende con este estudio ofrecer a los profesionales de la intervención nuevos datos que permitan mejorar su actuación con las familias y proporcionar mayor eficacia en sus actuaciones.

Palabras clave: familia; inestabilidad familiar; satisfacción familiar; estrés; comunicación; recursos; inteligencia emocional

\section{ABSTRACT}

Family satisfaction, comunication and emotional intelligence.

In general, for the development of people and society, the family institution is a fundamental pillar. One of the most important social motivations of the human being developed during childhood is the affiliation: maintain positive affective relationships with other people. In addition, one of the vital affections in the human being is the couple's relationship since it allows satisfying basic needs and in turn establishing patterns of development and behavior.

Based on the model of family instability proposed by Rodríguez, Rodríguez-Mateo and Luján (2015), the present work has several objectives: first, to know if the family variables interrelated in said model (family stress, family communication, family resources, family satisfaction and marital instability) can be related to a new variable (Emotional Intelligence); and secondly, to obtain possible influences and interactions, trying to replicate the model proposed by said authors.

This research is focused on the search of the different degrees of association or relationship that exists between a relevant group of variables present in the family environment with satisfaction and marital instability. This set of variables related to the family life cycle (family communication, family resources, family stress) directly affect family relationships and is associated with different degrees of well-being in families. In addition, a new variable has been added with respect to the 
study on the model of family instability in the breakup of a partner (Rodríguez, Rodríguez-Mateo and Luján, 2015), emotional intelligence, to verify its relationship with marital stability. Through different scales we want to evaluate these relationships and check whether these factors (stress, communication, resources and emotional intelligence) have a direct impact on family satisfaction and stability.

The instruments used in the present research work, to operationalize the variables to be studied, have been compiled from the Manual of Family Assessment Instruments (2008) published by the University of Deusto and the Scale of Self-Reported Emotional Intelligence (TMMS-24) of Extremera and Fernández-Berrocal (2004).

The sample corresponds to a group of 96 people, whose inclusion criteria were family group membership and no cognitive difficulty to perform the questionnaires that are attached.

The data analysis techniques used have been the correlational analysis between family variables, multiple linear regression, structural model proposal analysis through the confirmation of relationships between variables (confirmatory model), and confirmatory factor analysis.

Two factors were observed, total communication and total resources, as predictors of total satisfaction. It has been possible to obtain a confirmatory model that explains the variables associated with family satisfaction through the AMOS program. It is still postulated, through confirmatory factor analysis, the variable family experience as "injector" of the other variables.

The results support a new explanatory model of relationship between these variables and confirm the predictive power of communication and family resources in family satisfaction.

The aim of this study is to offer intervention professionals new information to improve their performance with families and to provide greater efficiency in their actions.

Keywords: family; family instability; family satisfaction; stress; communication; resources; emotional intelligence

\section{INTRODUCCIÓN}

\section{Satisfacción familiar y marital.}

La familia como constructo social es una de las más significativas acciones desarrolladas por el ser humano para expresar de manera libre su yo interior, sus emociones y sentimientos, sus valores y creencias, en un entorno íntimo y cercano, con el objetivo de ser feliz en interacción con otro ser humano y que permite el crecimiento personal y el desarrollo de su madurez beneficiándose del bienestar resultante. De esta manera, Clavijo (2002) afirma que no hay vínculo más importante para el ser humano que aquel que necesita y adquiere muy pronto en su medio familiar y que mantiene a lo largo de todo su desarrollo vital.

A lo largo de la historia han existido muchos cambios en la sociedad que han provocado una modificación en el papel que ejercen las familias, aunque siga siendo transmisora de valores, hábitos y costumbres, así como el principal apoyo emocional y económico (Cendrero, 2006). En la actualidad, la familia ha evolucionado hacia una gran variedad de tipologías. Para Rodrigo y Palacios (2005) se trata de una unión de personas que comparten un propósito vital de existencia en común que se prevé duradero, en el que existen fuertes sentimientos de pertenencia a ese grupo, donde hay compromiso personal entre ellos y se construyen intensas relaciones de intimidad, reciprocidad y dependencia. Escrivá y Montoro (2011) argumentan que el amor, el afecto y la ternura, en cuanto a fines de la relación de la pareja, fluctúan a lo largo del tiempo por lo que no son suficientes para crear lazos duraderos.

La satisfacción familiar es de vital importancia para que la familia pueda mantener un recorrido duradero, para que pueda llevar a cabo sus funciones como sistema social y para que pueda servir 


\section{SATISFACCIÓN FAMILIAR, COMUNICACIÓN E INTELIGENCIA EMOCIONAL}

de modelo saludable para su descendencia y entorno. En este sentido, Bernal (2008) afirma que la insatisfacción emocional no solo afecta al entorno familiar, sino que se generaliza a diversos aspectos de la vida de las personas como son las relaciones sociales, las relaciones de trabajo, el rendimiento laboral y la salud física.

La satisfacción marital, en cambio, se refiere al grado de satisfacción entre una pareja, es decir, el grado de satisfacción que ellos sienten con su relación (Edalati y Redzuan, 2010). Asi, Fontana y Fernández (2011) definen la satisfacción conyugal como la naturaleza y peculiaridades de una relación de pareja que presenta bienestar psicológico y donde existen patrones de interacción en las principales áreas de la vida marital y en los diferentes niveles cognitivos, afectivos y comportamental. Por tanto, la satisfacción marital se refiere al grado de satisfacción que existe entre una pareja, lo que ellos sienten con su relación (Flores, 2011).

\section{La comunicación familiar.}

Para todo ser humano la capacidad de comunicarse se torna como un elemento principal para lograr su desarrollo dentro de la sociedad en que se desenvuelve. La comunicación permite la expresión de emociones, sentimientos, ideas, metas, ilusiones, etc. es lo que le ayuda a iniciar con las personas que le rodean relaciones de tipo íntimo (Pérez y Estrada, 2006). La comunicación es un canal por el cual se desea manifestar y transmitir alguna idea. Toda comunicación implica un compromiso y, por ende, define la relación. Pero no sólo transmite mera información, sino que, al mismo tiempo impone ciertas conductas (Watzlawick, 2008). Franco (1995), citado en Pérez y Estrada (2006), sostiene que nos realizamos como personas en la medida en que nos comunicamos.

Para Pearson y Sessler, (1991) la comunicación en una familia es como una fuerza dinámica y primordial en el mantenimiento de las relaciones. Mientras sus miembros manifiesten comprenderse y soportarse, cierta estabilidad y autonomía, resolver los conflictos, tomar decisiones y manejar el poder y el estrés, la comunicación facilita el desarrollo de la satisfacción y la salud familiar.

\section{Los recursos familiares, estrés e inestabilidad familiar.}

Los recursos familiares no sólo hacen referencia a los aspectos materiales y/o económicos que posee una familia, sino que engloba a todos aquellos vínculos, flexibilidad, una comunicación abierta que a su vez contribuyen a desarrollar los recursos personales en cada uno de sus miembros. Martínez y Méndez (1997) afirman que los recursos familiares son las fuentes de apoyo intra o extra familiares que pueden utilizarse en la solución de los problemas que se presentan en la familia. Los recursos pueden ser o bien sociales, o culturales, religiosos, educativos, económicos o médicos.

Las familias se enfrentan continuamente a diferentes situaciones en las que tienen que lidiar con innumerables circunstancias que pueden provocar cierto nivel de estrés en ellos. Ciertos niveles de estrés pueden ser adecuados, si nos ayudan a afrontar situaciones de nuestra vida diaria. Pero el problema surge cuando las personas se sienten incapaces de responder ante tales circunstancias. La relación individuo-ambiente será o no estresante en función de la evaluación cognitiva del sujeto. Se podría decir que el valor estresante de una situación va a depender de cómo lo perciba cada persona. De tal forma que, una situación puede ser o no estresante para ciertas personas (González y Lorenzo, 2012).

López (2002) refiere que las familias inestables son fuente de desequilibrios que sufren graves consecuencias personales y relacionales, mientras que las familias estables establecen vínculos afectivos sólidos, virtudes y estímulos que mejoran la condición de los miembros familiares en su consideración individual y social.

En la investigación realizada por Rodríguez, Rodríguez-Mateo y Luján (2015), se observa la relación existente entre las variables familiares tales como la comunicación familiar, el estrés fami- 
liar, los recursos familiares en relación con la satisfacción familiar y la inestabilidad matrimonial. Los profesores Rodríguez-Mateo y Luján habían realizado distintas aproximaciones a las variables familiares, la negociación y la mediación (Perez y Rodríguez-Mateo, 2014; Rodríguez-Trueba, RodríguezMateo y Luján, 2014; Santana y Rodríguez-Mateo, 2014; Díaz-Negrín, Rodríguez-Mateo, RodríguezTrueba y Luján, 2015; Luján, Rodríguez-Mateo y Rodríguez Trueba, 2015; Díaz-Negrín, Luján, Rodríguez-Mateo y Rodríguez-Trueba, 2015).

La investigación realizada por Rodríguez, Rodríguez-Mateo y Luján, tenía como objetivo principal demostrar si existe relación entre determinadas variables familiares seleccionadas, teniendo en cuenta dos modelos que han trabajado la influencia de factores en la inestabilidad familiar y de ruptura familiar: el modelo MASH de Olson y el modelo en cascada de Gottman. Con la finalidad de confirmar si los factores de comunicación, estrés y recursos familiares inciden sobre la satisfacción, y la inestabilidad familiar con la satisfacción. Se confirmó que estas relaciones son intensas y se diseñó un modelo estructural que explicaría esas variables asociadas a la inestabilidad de la pareja como predictoras del divorcio. Se pudo llegar a hipotetizar un modelo que explicaría las variables asociadas a la inestabilidad matrimonial como predictoras del divorcio.

A partir de todos los datos y resultados de dicha investigación, se establece para la producción del presente trabajo un punto de partida tomando como referencia todos ellos para refutar dichos datos, elaborar las diferentes hipótesis planteadas y proponer, si fuera el caso, un nuevo modelo.

\section{La inteligencia emocional}

La IE puede ser entendida de diferentes maneras, teniendo en cuenta el enfoque de cada uno de los autores y el seguimiento de uno u otro modelo. Salovey y Mayer (1997), citado en Extremera y Fernández-Berrocal (2004), describen la Inteligencia Emocional como la habilidad para percibir, valorar y expresar la emoción adecuadamente y adaptativamente; la destreza para permitir y/o generar sentimientos que faciliten el pensamiento; la habilidad para comprender las emociones y la cognición emocional; y la práctica para regular las emociones para originar el crecimiento intelectual y emocional.

Diversos estudios han relacionado la inteligencia emocional con diferentes ámbitos como el estrés ocupacional, el ajuste psicológico y emocional, la percepción de satisfacción con la vida y la calidad de las relaciones interpersonales y también con el éxito académico (Bermúdez, Álvarez y Sánchez, 2003). Martínez-Pons (1997), citado en Bermúdez, Álvarez y Sánchez (2003), encontró una relación positiva entre inteligencia emocional, satisfacción con la vida y la subescala de dominio de tareas. Por otra parte, Schutte et al. (2001), citado en Bermúdez, Álvarez y Sánchez (2003), llevó a cabo siete estudios en los que analizó la relación entre inteligencia emocional y relaciones interpersonales. Se encontró correlación positiva entre inteligencia emocional, empatía y autocontrol en las situaciones sociales y las relaciones afectivas, y más respuestas de cooperación hacia sus parejas. Además, cuando se evalúa la inteligencia emocional como alta en sus parejas, se demuestra que tienen una relación de pareja más satisfactoria. A esta misma conclusión llegó Fitness (2001), citado en Bermúdez, Álvarez y Sánchez (2003), que encontró que las personas que saben percibir, identificar y expresar emociones tienen unas relaciones de pareja más felices.

Es posible, por tanto, que pueda existir una relación positiva entre inteligencia emocional y la estabilidad emocional y familiar. En consecuencia, uno de los objetivos de la presente investigación es analizar la relación entre las distintas variables mencionadas y la inteligencia emocional.

\section{OBJETIVOS DE LA INVESTIGACIÓN.}

Este estudio tiene como objetivos los siguientes: 


\section{SATISFACCIÓN FAMILIAR, COMUNICACIÓN E INTELIGENCIA EMOCIONAL}

Conocer las relaciones entre satisfacción familiar y las variables estrés familiar, comunicación, recursos familiares e inteligencia emocional.

Obtener una función de regresión que pueda predecir la puntuación de la satisfacción familiar (tomada como variable dependiente) a partir de las puntuaciones en el resto de las variables (variables independientes).

Obtener un modelo que explique las variables asociadas a la satisfacción familiar.

Comprobar las posibles diferencias de género en relación con las variables comentadas.

Poner a prueba la posibilidad de la existencia de un componente principal que explique el funcionamiento familiar.

\section{MÉTODO}

\section{Muestra y/o participantes.}

La muestra corresponde a un grupo de 96 personas, cuyos criterios de inclusión fueron la pertenencia a grupo familiar y sin dificultad psíquica para poder realizar los cuestionarios que se anexan. Se entiende por grupo familiar donde no hay individuos independientes con estas características. Por ejemplo, parejas que conviven, familia casada con hijos o sin hijos y así tantas opciones como plural es la sociedad en la que vivimos.

Por otra parte, la selección no ha sido mediante muestreo aleatorio, pues se pretendía acceder a un grupo significativo específico, personas con relaciones estables o matrimonios, con hijos o sin hijos. Se contó con un total de 96 personas, que han participado voluntariamente luego de solicitar su colaboración, de las cuales 66 mujeres y 30 hombres.

\section{Procedimiento, variables e instrumentos utilizados}

Para la obtención de los datos necesarios para esta investigación, se ha aplicado seis cuestionarios de forma personal y no presencial a las 96 personas que han sido partícipes en la presente investigación. Se les explica la importancia de recopilar estos datos y obtener información acerca de la satisfacción familiar, la comunicación, los recursos, el estrés, la inestabilidad familiar y por último, la inteligencia emocional.

Las variables a estudiar son la satisfacción familiar, la comunicación familiar, los recursos familiares, el estrés familiar, la inestabilidad matrimonial, y la inteligencia emocional.

Los instrumentos utilizados en el presente trabajo de investigación, para operativizar las variables a estudiar, han sido recopilados del Manual de Instrumentos de evaluación familiar (2008) publicado por La Universidad de Deusto y la Escala de Inteligencia Emocional Autoinformada (TMMS-24) de Extremera y Fernández-Berrocal (2004).

Escala de Satisfacción Familiar (FSfS. Family Satisfaction Scale). Evalúa la satisfacción que experimenta una familia sobre su nivel de cohesión y adaptabilidad familiar. Es original de Olson, y Wilson, (1982) adaptada al español por Sanz (2003) y Iraurgi, y Martínez-Pampliega, (2002). Presente unos índices de fiabilidad de 0,92 y 0,95 alpha de Cronbach y test-pretest de Pearson respectivamente.

Escala de comunicación Familiar (FCS, Family Communication Scale). Evalúa la comunicación que se origina en la familia, de Barnes y Olson (1982) y ha sido adaptada al español por Sanz (2003) y por Sanz, Iraurgi y Martínez-Pampliega (2002). Presenta una fiabilidad con alpha de Cronbach de .88. Presenta una adecuada estabilidad temporal siendo la correlación test-retest e intraclase de .88.

Escala de recursos familiares (Family Strenghs). Evalúa los recursos con los que cuenta una familia. Es original de Olson, Larsen y McCubbin (1982) y ha sido adaptada a España por Sanz (2003) y por Sanz, Iraurgi y Marftínez-Pampliega (2002). La fiabilidad de la escala total en la adaptación española ha sido de un alpha de Cronbach de .85 . 
Escala de estrés familiar (Family Stress). Evalúa el nivel de estrés familiar. Original de Olson (1992) y adaptada a España por Sanz (2003) y por Sanz, Iraurgi y Martínez-Pampliega (2002). Este instrumento presenta una fiabilidad alta en la escala global (alpha de Cronbach de 0,85) en Olson (1997) y una fiabilidad de 0,82 en Sanz (2003).

Escala de Inestabilidad Matrimonial (MIS. Marital Instability Scale). Evalúa la inestabilidad entre las parejas intactas dirigida a predecir la ruptura matrimonial, es decir, la tendencia al divorcio, independientemente de la calidad matrimonial. Es aplicable solo a parejas casadas. Es original de Booth y Edwards (1983) y ha sido adaptado por Martínez-Pampliega, Sanz e Iraurgi (2002). La versión reducida de la escala presentó un índice de fiabilidad de 0,75.

Escala de Inteligencia Emocional Autoinformada (TMMS-24) de Extremera y FernándezBerrocal (2004). La escala TMMS-24 contiene tres dimensiones claves de la IE con 8 ítems cada una de ellas: Atención Emocional, Claridad de Sentimientos y Reparación Emocional. Consta de 24 ítems que se evalúan en un formato tipo Likkert con cinco alternativas de respuesta. $1=$ Nada de acuerdo hasta 5 = Totalmente de acuerdo. Fernández y Extremera, (2004), presentan índices de consistencia interna de 0.90 para Atención Emocional, 0.90 para Claridad Emocional y 0.86 para Reparación Emocional.

\section{RESULTADOS}

\section{Análisis correlacional}

La satisfacción familiar se correlaciona con las variables comunicación, recursos, estrés, inestabilidad e inteligencia emocional reparación y los datos resultantes se reflejan en la Tabla 2:

Tabla 1: Análisis correlacional entre las variables familiares.

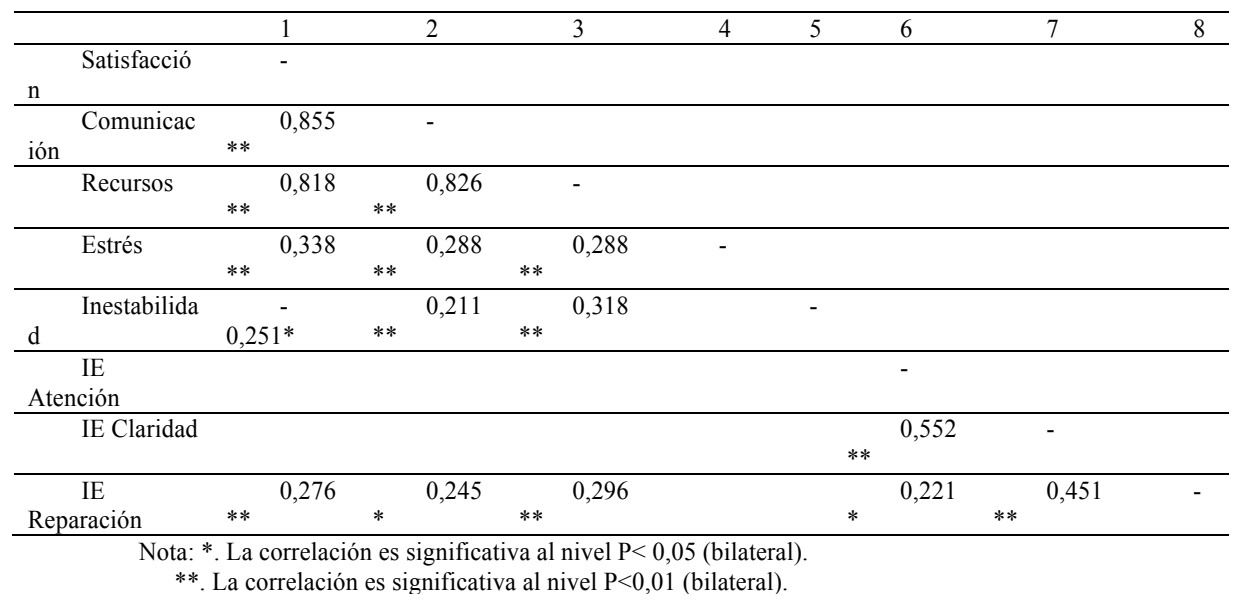

\section{Regresión lineal}

Se realiza una regresión lineal tomando como variable dependiente la satisfacción familiar, y como variables independientes o predictoras el resto de las variables.

Se observó dos factores, la comunicación total y los recursos totales como variables predictoras 0 independientes y como variable dependiente la satisfacción total. Se utilizó el método por pasos. Se observa en las tablas número 3,4 y 5 : 


\section{SATISFACCIÓN FAMILIAR, COMUNICACIÓN E INTELIGENCIA EMOCIONAL}

Tabla 2: Resumen del modelo

\begin{tabular}{ccccc}
\hline Modelo & $\mathrm{R}$ & R cuadrado & R cuadrado & Error estándar de la \\
\hline 1 & & & ajustado & estimación \\
\hline 2 & $\begin{array}{c}0,835 \\
\text { a }\end{array}$ & 0,697 & 0,694 & 3,40872 \\
\hline $\begin{array}{c}0,866 \\
\text { b }\end{array}$ & 0,750 & 0,744 & 3,11721 \\
\hline
\end{tabular}

1. Predictores: (Constante), COMTOT

2. Predictores: (Constante), COMTOT, RECTOT

Nota: El modelo propuesto, y en concreto el coeficiente de determinación (R cuadrado ajustado) explica el 74\% de la varianza.

\section{Modelo de ecuación estructural}

A través de los datos obtenidos se podría tener una hipótesis de un modelo que explica las variables asociadas a la satisfacción familiar. Para ello y a través del programa AMOS, versión 23 se plantea el modelo y se somete a comprobación. El modelo propuesto es el que se plantea en la figura 1.

Figura 1:

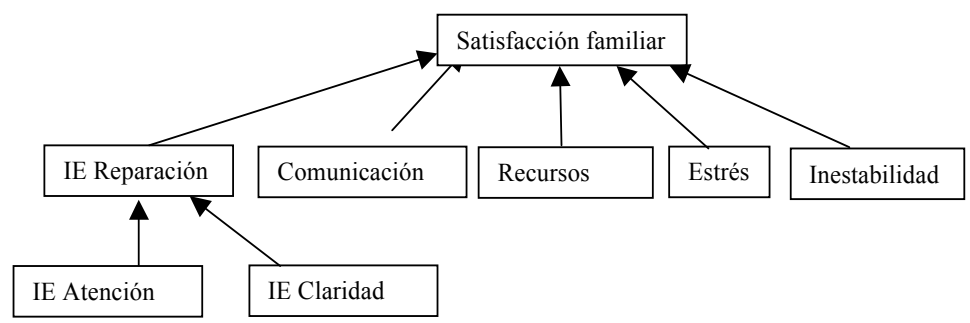

Los indicadores de bondad de ajuste analizados son los siguientes:

-CFI (Comparative Fit Index). Se considera que debe estar en torno a 0,95 para considerar que el modelo se ajusta adecuadamente a los datos. En este caso el programa arroja un CFI igual a 0,934 .

-RMSEA (Root Mean Square Error of Approximation) o error de aproximación. El dato RMSEA es 0,095 con lo que no se rechaza y nos permite aceptarlo.

-Chi Cuadrado. Este dato 1,9186 es aceptable ya que es inferior a 2.

A la vista de estos resultados se puede aceptar este modelo como propuesta teórica consistente.

\section{T-Test por sexo}

Se quiere comprobar si existen diferencias en las variables analizadas en este estudio en función del sexo. Para ello se realiza un análisis de diferencia de medias (T-Test) de todas las variables cuantitativas, en función de la variable cualitativa "sexo".

Salvo en una variable, no existen diferencias por sexo. Solamente se aprecia diferencia entre ambos sexos en la variable IE Atención. Aunque las estadísticas de grupo demuestran unas medias superiores en el caso de las mujeres e inferiores en el caso de los hombres, estas diferencias no son significativas, salvo en inteligencia emocional atención. 


\section{Análisis factorial confirmatorio}

Después de todos los datos obtenidos, se plantea una nueva hipótesis de un modelo que explique que todas las variables asociadas entre sí estén influenciadas por una variable oculta a la que Ilamaremos vivencia familiar. Para ello y a través del programa AMOS se plantea el modelo y se somete a su comprobación mediante análisis factorial confirmatorio. El análisis factorial confirmatorio es el que nos permite contrastar un modelo construido con antelación, en el cual el investigador instituye a priori el conjunto total de las relaciones entre los elementos que lo conforman. A diferencia del factorial exploratorio, en este se presupone que el investigador es capaz de aventurar con anterioridad la estructura de datos (preferiblemente en función de una teoría bien fundamentada) y sólo precisa corroborar que esa estructura planteada pueda también obtenerse empíricamente (Herrero, 2010). El modelo propuesto es el que se plantea en la figura 6:

Figura 2:

Modelo de vivencia familiar.

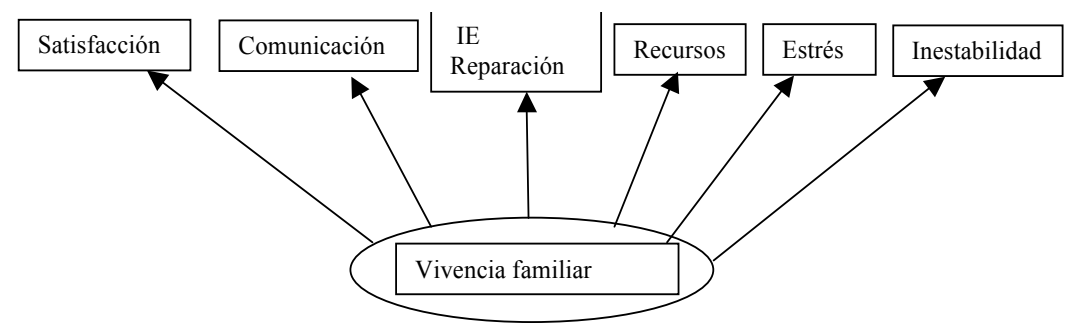

Los indicadores resultantes son los siguientes:

-CFI (Comparative Fit Index). El programa arroja un CFI de 1.

-RMSEA el dato RMSEA es 0,000 por lo que indica un buen ajuste de los datos.

-Chi Cuadrado. Los datos obtenidos de la chi cuadrado son: 0,7977. Razón entre chi cuadrado y los grados de libertad: $7,18 / 9=1,9186$, por lo que este dato 1,9186 es aceptable ya que es inferior a 2.

A la vista de estos resultados se puede confirmar que el modelo es bueno y que las variables asociadas están correlacionadas con esa variable oculta a la que hemos llamado vivencia familiar.

\section{DISCUSIÓN Y CONCLUSIONES}

El objeto de este trabajo está basado en analizar a determinados participantes que cumplieran unos requisitos en relación a determinadas variables relacionadas entre sí y a su vez, como se relacionaban con su estado de satisfacción familiar y mostraban inestabilidad matrimonial. Los criterios de selección fueron aquellos que delimitaban a un grupo de personas que fuesen matrimonios 0 parejas de hecho, con hijos o sin hijos. Esta delimitación podría paliar de alguna manera que el grupo no estuviera compuesto por pertenecientes a parejas ya divorciadas.

Se ha tomado como punto de partida la investigación propuesta por Rodríguez, RodríguezMateo y Luján (2015) para analizar las variables familiares en relación con la satisfacción familiar y la inestabilidad matrimonial a partir de otros criterios de selección, basándonos en otros modelos para su análisis y teniendo en cuenta una nueva variable (la inteligencia emocional). Mientras que dicha investigación utiliza una muestra de menor tamaño y con ciertas particularidades (cerca de los parámetros de divorcio, edad, situación laboral, media de número de año de casados, no de hijos) el presente trabajo utiliza mayor número de personas y un grupo significativo específico, personas 


\section{SATISFACCIÓN FAMILIAR, COMUNICACIÓN E INTELIGENCIA EMOCIONAL}

con relaciones estables o matrimonios, con hijos 0 sin hijos. Los resultados de ambas investigaciones mantienen aspectos comunes tales como, la fuerte relación entre la satisfacción familiar con las variables comunicación y recursos y la correlación negativa con el estrés familiar. Además, el modelo propuesto en ambas investigaciones arroja unos indicadores de bondad que se ajustan a los indicadores propuestos. En esta investigación se ahonda en otros aspectos incluyendo una nueva variable y presentando otras hipótesis con nuevos modelos.

En relación a los resultados obtenidos de la presente investigación para la muestra analizada podemos decir lo siguiente:

La comunicación familiar, los recursos familiares y la IE Reparación se correlacionan positivamente con satisfacción familiar y estrés familiar se correlaciona negativamente con satisfacción familiar, al igual que la inestabilidad matrimonial. Martínez (1995) refiere que una comunicación inefectiva afecta a la relación de pareja cuando existen conflictos, sentimientos de insatisfacción y afecta a su vez a la estabilidad marital. Por otra parte, Bradbury y col (1998), citado en Fincham y Beach (1999) señalan que altos niveles de estrés generan patrones de comunicación negativos y menor satisfacción matrimonial.

-Además, se puede observar como existe un elemento oculto que influye directamente sobre todas las variables y que correlacionan entre sí.

-La inteligencia emocional está relacionada con la satisfacción familiar, sobretodo en mayor medida la reparación, siendo en un alto porcentaje en las mujeres. Las otras dos, la claridad y atención se correlacionan entre ellas pero no con el resto de las variables. Por lo que podemos decir que la inteligencia emocional no influye directamente en la inestabilidad matrimonial.

-El sexo influye en la satisfacción familiar y en la inestabilidad matrimonial, donde se ha podido observar unos resultados de media superiores en el caso de la mujer y unos datos inferiores en el caso de los hombres.

Por tanto, se podría decir que se reformula la anterior investigación propuesta por Rodríguez, Rodríguez-Mateo y Luján, puesto que existe una nueva propuesta que influye en dichas variables, como la relación de la inteligencia emocional en la satisfacción familiar, y el cambio de la influencia de la variable inestabilidad familiar, que pasa a ser un elemento más que influye en la satisfacción familiar. Se confirma asimismo la vivencia familiar como un factor oculto que influye en todas las variables estudiadas.

Finalmente, podemos decir que existen algunas definiciones de autores citados en esta investigación que no se alejan de la realidad teniendo en cuenta los resultados obtenidos, como por ejemplo:

La satisfacción marital se refiere al grado de satisfacción entre una pareja, es decir, el grado de satisfacción que ellos sienten con su relación (Edalati y Redzuan ,2010). Si bien es cierto que deben poseer un alto grado de satisfacción también deben de tener otros aspectos fundamentales que sean de igual importancia que la propia satisfacción.

Para Pearson y Sessler, (1991) la comunicación en una familia es como una fuerza dinámica y primordial en el mantenimiento de las relaciones. Mientras sus miembros manifiesten comprenderse y soportarse, cierta estabilidad y autonomía, resolver los conflictos, tomar decisiones y manejar el poder y el estrés, la comunicación facilita el desarrollo de la satisfacción y la salud familiar.

Esta definición de Pearson y Sessler confirma con claridad los resultados obtenidos con respecto a esta variable, puesto que la comunicación es fundamental para que haya un buen mantenimiento de las relaciones y vemos como su importancia radica en que a mejor comunicación existente, mejores decisiones se pueden tomar y así poder manejar el poder y el estrés, puesto que sirve de canal para que haya buena satisfacción familiar. 
Estos datos pueden ser sugerencias de utilidad para la intervención familiar. A través del modelo de ecuación estructural propuesto se podría conocer la importancia de la comunicación que influye directamente con todos las variables utilizadas para reconocer el papel primordial de la misma y buscar nuevos puentes de comunicación en las relaciones de pareja. A su vez, la importancia que ejerce la existencia del estrés familiar, puesto que todos los resultados arrojan que a mayores niveles estresores hay en la familia, menor es la satisfacción familiar y mayor la inestabilidad matrimonial.

Algunas limitaciones que se podría observar es que, por un lado, la muestra no es igualitaria en el número de hombres y de mujeres, por tanto podría existir margen de error y ausencia de datos y comparaciones exactas entre ambos sexos. Por otro lado, se podría haber establecido diferencias entre los grupos de participantes, es decir, analizar casos de parejas ya divorciadas o separadas y establecer diferencias entre ambos grupos. A su vez, analizar matrimonios con hijos y por otra parte matrimonios sin hijos, y así establecer sus diferencias.

\section{REFERENCIAS BIBLIOGRÁFICAS.}

Bermúdez, M., Álvarez T., Sánchez A. (2003). Análisis de la relación entre inteligencia emocional, estabilidad emocional y bienestar psicológico. Red de revistas científicas de América Latina y el Caribe, España y Portugal, 2(001) 27-32. Recuperado de http://estadisticasoctys.freetzi. com/Estadistica1/Estadisticadescriptiva/LectCorrelacion.pdf

Bernal, T. (2008). La mediación, una solución a los conflictos de ruptura de pareja. Madrid, España: Colex.

Cendrero, T. (2006). Mediación familiar: experto en gestión de conflictos. Jaén, España: Formación Alcalá.

Clavijo, A. (2002). Crisis, familia y psicoterapia. Recuperado de http://newpsi.bvs-psi.org.br/ebooks2010/en/Acervo_files/Crisisfamiliapsicoterapia.pdf

Díaz-Negrín, M.; Luján, I.; Rodríguez-Mateo, H. y Rodríguez-Trueba, J.C. (2015). Análisis de la gestión e implementación en mediación en el ámbito escolar. Internacional Journal of Developmental and Educational Psychology,1 (1) ,275-284. Recuperado de http://www.infad.eu/RevistaINFAD/OJS/index.php/IJODAEP/article/download/108/129.

Díaz-Negrín, M.; Rodríguez-Mateo, H.; Rodríguez-Trueba, J.C. y Luján, I. (2015). Valor predictivo de variables socioafectivas en mediadores escolares. Internacional Journal of Developmental and Educational Psychology, 1 (1), 265-274. Recuperado de http://infad.eu/RevistalNFAD/OJS/index.php/IJODAEP/article/view/113/128

Edalati, A. \& Redzuan, M. (2010). A Review: Dominance, Marital Satisfaction and Female Aggression. Journal of Social Sciences. 6 (2), 162-166. Recuperado de http://thescipub.com/PDF/jssp.2010.162.166.pdf

Escrivá, J., y Montoro, C. (2011). Relaciones de pareja. Un análisis de la realidad española a partir de la encuesta "la familia, recurso de la sociedad". Recuperado de file:///C:/Users/Yurena/Downloads/30430_Escriva-Montoro_Familia-2012\%20(3).pdf

Extremera, N. y Fernández-Berrocal, P. (2004). El uso de las medidas de habilidad en el ámbito de la inteligencia emocional. Ventajas e inconvenientes con respecto a las medidas de auto-informe. Boletín de Psicología, 80, 59-77. Recuperado de http://www.uv.es/seoane/boletin/previos/N80-3.pdf

Extremera y Fernández Berrocal (2004) Escala de Inteligencia Emocional Autoinformada (TMMS24). Recuperado de http://www2.uned.es/competencias-emocionales/TMMS24\%20con\% 20referencias.pdf 


\section{SATISFACCIÓN FAMILIAR, COMUNICACIÓN E INTELIGENCIA EMOCIONAL}

Flores, M. (2011). Comunicación y Conflicto: ¿Qué Tanto Impactan en la Satisfacción Marital? Acta de investigación psicológica, 1 (2), 216-232. Recuperado de http://www.scielo.org.mx/pdf/aip/v1n2/v1n2a3.pdf

Fontana, M., y Fernández, M. (2011). Evaluación de la satisfacción conyugal e interacción padreshijos en familias de la comunidad de Madrid. International Journal of Developmental and Educational Psychology, 2(1), 511-520. Recuperado de http://infad.eu/RevistalNFAD/ 2011/n1/volumen2/INFAD_010223_511-520.pdf

González, I., y Lorenzo, A. (2012). Propuesta teórica acerca del estrés familiar. Revista electrónica de Psicología Iztacala, 15(2), 416-432. Recuperado de http://www.iztacala.unam.mx/carreras/ psicologia/psiclin/vol15num2/Nol15No2Art5.pdf

López, M. (2002). Protección jurídica de la estabilidad familiar. Anales de derecho (20), 55-68. Recuperado de http://revistas.um.es/analesderecho/article/viewFile/56741/54691

Martínez, F. y Méndez, E. (1997). Recursos familiares. Garza ET. Trabajo con familias. México: Universidad Autónoma de Nuevo León. 107-111. Recuperado de http://www.facmed.unam.mx /deptos/familiar/compendio/Segundo/II_EMF_221-224.pdf

Pearson, J.; Sessler,C. (1991). Family Communication and Health: Maintaining Marital Satisfaction and Quality of Life. Annual Meeting of the International Communication Association. 2-28. Recuperado de http://files.eric.ed.gov/fulltext/ED335722.pdf

Pérez, G. y Estrada, S. (2006). Intimidad y comunicación en cuatro etapas de la vida de pareja: su relación con la satisfacción marital. Archivos Hispanoamericanos de Sexología, 12 (2), 133-163. Recuperado de http://biblio.upmx.mx/Estudios/Documentos/matrimonio0116.asp

Pérez, V y Rodríguez-Mateo, H (2014). Mediación Familiar y adolescencia. Internacional Journal of Developmental and Educational Psychology, 1 (6) ,197-206.

Rodrigo, M. y Palacios, J. (2005). Familia y desarrollo humano. Madrid, España: Alianza.

Rodríguez, A.; Rodríguez-Mateo, H. y Luján, I. (2015). Modelo de inestabilidad familiar en la ruptura de pareja. International Journal of Developmental and Educational Psychology INFAD Revista de Psicología, 1 (2), 91-102. Recuperado de http://www.infad.eu/RevistalNFAD /OJS/index.php/IJODAEP/article/view/111/54

Rodríguez-Trueba, J.C.; Rodríguez-Mateo, H. y Luján, I. (2014). Validez Predicitiva del pensamiento constructivo en la eficacia negociadora. Internacional Journal of Developmental and Educational Psychology 1 (6), 245-258.

Santana, G. y Rodríguez-Mateo, H. (2014). El perfil de eficacia del mediador desde la perspectiva de la negociación. Internacional Journal of Developmental and Educational Psychology, 1 (6), 237244.

Watzlawick, P. (2008). Teoría de la comunicación humana. Interacciones, patologías y paradojas. Barcelona, España: Herder. 